\title{
Explicit form of Pauli potential for direct derivation of pair density from a two-particle differential equation for the quintet state of four electrons with harmonic interparticle interactions
}

\author{
A. Akbari ${ }^{a, b}$, C. Amovillic ${ }^{c *}$ N. H. March ${ }^{d, e}$ and A. Rubio ${ }^{a, b}$ \\ a Departamento de Fisica de Materiales, Facultad de Químicas, \\ UPV/EHU, Apdo 1072, 20080 San Sebastián, Spain \\ ${ }^{b}$ Donostia International Physics Center, \\ Paseo Manuel de Lardizabal 4, 20018 San Sebastián, Spain \\ ${ }^{c}$ Dipartimento di Chimica e Chimica Industriale, \\ Università di Pisa, Via Risorgimento 35, 56126 Pisa, Italy \\ E-mail:amovilli@dcci.unipi.it \\ $d$ Department of Physics, University of Antwerp, \\ Groenenborgerlaan 171, B-2020 Antwerpen, Belgium \\ e Oxford University, Oxford, England
}

January 21, 2013

\begin{abstract}
Recently, an analytic two-particle density matrix (2DM) has been derived for the quintet state of four electrons interacting via two-body harmonic forces. Here we use this $2 \mathrm{DM}$ to extract the exact pair density $\Gamma\left(\mathbf{r}_{1}, \mathbf{r}_{2}\right)$. This is then employed in the known two-particle partial differential equation for the pair density amplitude to extract the Pauli potential $v_{P}\left(\mathbf{r}_{1}, \mathbf{r}_{2}\right)$ for this quintet state.
\end{abstract}

${ }^{*}$ Corresponding author. 


\section{Theory and results}

A variety of uses have now been made of the Pauli potential $v_{P}(\mathbf{r})$ of conventional density functional theory (DFT) [1], as reviewed recently by one of us [2]. However, what is still lacking is the desired functional form $v_{P}[n]$, where the ground-state density $n(\mathbf{r})$ is, of course, the main tool of DFT.

More recently, some interest has been focussed on the pair density functional description [3,4]. There, a generalized Pauli potential has been defined in order to write a two-particle differential equation for the pair density amplitude, say $\chi\left(\mathbf{r}_{1}, \mathbf{r}_{2}\right)$, in an N-electron problem $[3,5]$.

But progress on the form of $v_{P}\left(\mathbf{r}_{1}, \mathbf{r}_{2}\right)$ to enable the direct derivation of $\chi\left(\mathbf{r}_{1}, \mathbf{r}_{2}\right)$ from such a two-particle differential equation has proved to be slow. Therefore, in this note, we shall utilize the work of Akbari et al [6], in which a model analytical two-particle density matrix $\Gamma\left(\mathbf{r}_{1}, \mathbf{r}_{2} ; \mathbf{r}_{1}^{\prime}, \mathbf{r}_{2}^{\prime}\right)$ was presented for four electrons in the quintet state interacting via two-body harmonic forces. The diagonal element of this $\Gamma$ is, of course, the desired pair density $\Gamma\left(\mathbf{r}_{1}, \mathbf{r}_{2}\right)$ which has a simple, translationally invariant form $\Gamma\left(r_{12}\right), r_{12}$ being the distance $\left|\mathbf{r}_{1}-\mathbf{r}_{2}\right|$, which is a product of $r_{12}^{2}$ and a Gaussian factor having also the argument proportional to $r_{12}^{2}$.

Below, using this exactly known model form, we shall extract the form of the Pauli potential, namely $v_{P}\left(\mathbf{r}_{1}, \mathbf{r}_{2}\right)$, from the above form of $\Gamma\left(r_{12}\right)$ using the two-particle differential equation given by Nagy and Amovilli [3]. This takes the explicit form, in atomic units $(\hbar=1, m=1)$, for the four-particle model under discussion:

$$
\left.\left.\left\{-\frac{1}{2}\left[\nabla_{1}^{2}+\nabla_{2}^{2}\right]+\frac{3}{2} \kappa r_{12}^{2}+v_{P}\left(\mathbf{r}_{1}, \mathbf{r}_{2}\right)\right\} \chi \mathbf{r}_{1}, \mathbf{r}_{2}\right)=\mu \chi \mathbf{r}_{1}, \mathbf{r}_{2}\right)
$$

We remind that in this equation the external potential has been set to zero by taking the limit of the external harmonic force constant $k_{\text {ext }}$ to $0^{+}$.

This is the point at which we must be quite explicit about the way the pair density $\Gamma\left(\mathbf{r}_{1}, \mathbf{r}_{2}\right)$ to be utilized below is derived. The origin is an admittedly somewhat simplicistic model due to Bruch [7]. This author considers four electrons moving under the influence of a central pairwise interaction $v\left(r_{i j}\right)$ given by

$$
v\left(r_{i j}\right)=\frac{1}{2} \kappa r_{i j}^{2}
$$

In the following discussion of the Pauli potential $v_{P}\left(\mathbf{r}_{1}, \mathbf{r}_{2}\right)$, we shall restrict ourselves to the quintet state, $S=2$, for which the exact wave function $\Psi^{(2 S+1)}=\Psi^{(5)}$ is known from the work of Bruch [7]. The form of $\Psi^{(5)}$ is then given in this reference as the product of a spatial wave function quoted below, and a spin function. We shall work below with 
the reduced spatial density matrices $\Gamma_{n}$ of Löwdin [8], to be derived from Bruch spatial wave function $\Psi^{(5)}\left(\mathbf{r}_{1}, \mathbf{r}_{2}, \mathbf{r}_{3}, \mathbf{r}_{4}\right)$ having the explicit, unnormalized form

$$
\Psi^{(5)}\left(\mathbf{r}_{1}, \mathbf{r}_{2}, \mathbf{r}_{3}, \mathbf{r}_{4}\right)=\mathbf{u} \cdot(\mathbf{v} \times \mathbf{w}) \exp Q
$$

Bruch gives the RHS of equation (3) quite explicitly by defining $\mathbf{u}$ to $Q$ in the following fashion [7]:

$$
\begin{aligned}
& \mathbf{u}=\frac{1}{2}\left(\mathbf{r}_{1}+\mathbf{r}_{2}\right)-\frac{1}{2}\left(\mathbf{r}_{3}+\mathbf{r}_{4}\right), \\
& \mathbf{v}=\mathbf{r}_{1}-\mathbf{r}_{2} \quad, \quad \mathbf{w}=\mathbf{r}_{3}-\mathbf{r}_{4},
\end{aligned}
$$

and

$$
Q=-\frac{\sqrt{\kappa}}{4}\left(2 u^{2}+v^{2}+w^{2}\right)
$$

Following, for instance, Dawson and March [9], one can reduce the Scrödinger equation (3) into an equation relating the reduced density matrices $\Gamma^{(3)}, \Gamma^{(2)}$ and $\Gamma^{(1)}$. In this way, Akbari et al [6] integrated $\Gamma^{(3)}$ to obtain the exact correlated $\Gamma^{(2)}$ as

$$
\begin{gathered}
\Gamma^{(2)}\left(\mathbf{r}_{1}, \mathbf{r}_{2} ; \mathbf{r}_{1}^{\prime}, \mathbf{r}_{2}^{\prime}\right) \propto\left\{\left(1-\frac{\sqrt{\kappa}}{4}\left|\mathbf{R}-\mathbf{R}^{\prime}\right|^{2}\right) \mathbf{r} \cdot \mathbf{r}^{\prime}+(\mathbf{R} \cdot \mathbf{r})\left(\mathbf{R} \cdot \mathbf{r}^{\prime}\right)+\left(\mathbf{R}^{\prime} \cdot \mathbf{r}^{\prime}\right)\left(\mathbf{R}^{\prime} \cdot \mathbf{r}\right)\right. \\
\left.-\left(\mathbf{R}^{\prime} \cdot \mathbf{r}\right)\left(\mathbf{R} \cdot \mathbf{r}^{\prime}\right)-(\mathbf{R} \cdot \mathbf{r})\left(\mathbf{R}^{\prime} \cdot \mathbf{r}^{\prime}\right)\right\} \exp \left[-\frac{\sqrt{\kappa}}{4}\left(\left|\mathbf{R}-\mathbf{R}^{\prime}\right|^{2}+r^{2}+r^{\prime 2}\right)\right] .
\end{gathered}
$$

Here $\mathbf{r}$ and $\mathbf{R}$ are defined by

$$
\mathbf{r}=\mathbf{r}_{1}-\mathbf{r}_{2} \quad, \quad \mathbf{R}=\frac{1}{2}\left(\mathbf{r}_{1}+\mathbf{r}_{2}\right)
$$

the primed quantities $\mathbf{r}^{\prime}$ and $\mathbf{R}^{\prime}$ being defined in a parallel manner from $\mathbf{r}_{1}^{\prime}$ and $\mathbf{r}_{2}^{\prime}$. Returning at the point to the pair density $\Gamma$ in equation (1), we have the definition

$$
\Gamma\left(\mathbf{r}_{1}, \mathbf{r}_{2}\right)=\Gamma^{(2)}\left(\mathbf{r}_{1}, \mathbf{r}_{2} ; \mathbf{r}_{1}, \mathbf{r}_{2}\right)
$$

and hence it follows almost immediately from equations (7) and (9) that the pair density is given by

$$
\Gamma\left(\mathbf{r}_{1}, \mathbf{r}_{2}\right) \propto\left|\mathbf{r}_{1}-\mathbf{r}_{2}\right|^{2} \exp \left(-\frac{\sqrt{\kappa}}{2}\left|\mathbf{r}_{1}-\mathbf{r}_{2}\right|^{2}\right)
$$


With the above background, it remains to extract the Pauli potential $v_{P}\left(\mathbf{r}_{1}, \mathbf{r}_{2}\right)$ from equations (1), (3) and (10) for the above quintet state of the Bruch model [7]. In terms of variables defined in equation (8), by writing

$$
\chi\left(\mathbf{r}_{1}, \mathbf{r}_{2}\right)=\Gamma^{1 / 2}(r) e^{i \mathbf{P} \cdot \mathbf{R}}
$$

which gives back $\Gamma$ as $\chi^{*} \chi$ and is perfectly consistent with our translationally invariant model example, we are then led, almost immediately to the equation defining $v_{P}\left(\mathbf{r}_{1}, \mathbf{r}_{2}\right)$ as

$$
\left[-\nabla^{2}+\frac{3}{2} \kappa r^{2}+v_{P}\right] \Gamma^{1 / 2}(r)=\mu_{0} \Gamma^{1 / 2}(r)
$$

Thanks to the particular form of the solution $\Gamma^{1 / 2}(r)$, it is a straightforward matter to perform the Laplacian differentiation on the LHS of the equation (12) and thereby, after some manipulation (see, for example, Makarewicz [2]), to extract the desired analytic form of the Pauli potential as

$$
v_{P}(r)=\frac{2}{r^{2}}-\frac{5}{4} \kappa r^{2}
$$

Moreover, $\mu_{0}$ turns out to be $(5 / 2) \kappa^{1 / 2}$. By way of example, we show in Fig. 1 the plot of $v_{P}(r)$ against $r$ for three cases corresponding to $\kappa=0.5,1$ and 2 atomic units.

In summary, we have used the Bruch model [7] with spatial wave function $\Psi^{(5)}$ quoted in equation (3) above, together with the Nagy and Amovilli [3] form of $v_{P}\left(r_{12}\right)$, to extract this Pauli potential in the exact analytical form (13). This, to our present knowledge, is the first time $v_{P}\left(r_{12}\right)$ has been calculated exactly as a simple route to the generation of the pair density. Of course, to complete the Nagy and Amovilli theory of the pair density amplitude $\chi$, we would eventually need $v_{P}[\Gamma]\left(\mathbf{r}_{1}, \mathbf{r}_{2}\right)$ rather than $v_{P}\left(r_{12}\right)$ calculated above for the Bruch model. In this particular case some progress in this direction through equations (10) and (13) is, in principle, achievable but with no claim that the resulting $v_{P}[\Gamma]\left(\mathbf{r}_{1}, \mathbf{r}_{2}\right)$ is the desired "universal functional".

\section{Acknowledgements}

A.A. to be added. C.A. aknowledges partial financial support from Italian Ministry of University and Research under the project PRIN 09. N.H.M. whishes to acknowledge that his contribution to this note was started during a visit to DIPC, San Sebastian. He thanks Professors P. Echenique and A. Rubio for generous hospitality. Also N.H.M. acknowledges continuing affiliation with Antwerp University through financial support kindly arranged by Professors D. Lamoen and C. Van Alsenoy. A.R. to be added. 


\section{References}

[1] R. G. Parr and W. Yang, Density-Functional Theory of Atoms and Molecules (Oxford University Press, New York, 1989).

[2] N. H. March, J. Mol. Structure (THEOCHEM) 943 (2010) 77.

[3] Á. Nagy and C. Amovilli, J. Chem. Phys. 121 (2004) 6640.

[4] Á. Nagy and C. Amovilli, J. Chem. Phys. 128 (2008) 114115.

[5] C. Amovilli and Á. Nagy, J. Chem. Phys. 129 (2008) 204108.

[6] A. Akbari, C. Amovilli, N. H. March and A. Rubio, Chem. Phys. Lett. 536 (2012) 162 .

[7] L. W. Bruch, J. Chem. Phys. 72 (1980) 5511.

[8] P. O. Loẅdin, Phys. Rev., 97 (1955) 1474.

[9] K. A. Dawson and N. H. March, J. Chem. Phys. 81 (1984) 5850.

[10] J. Makarewicz, Am. J. Phys. 54 (1986) 178. 


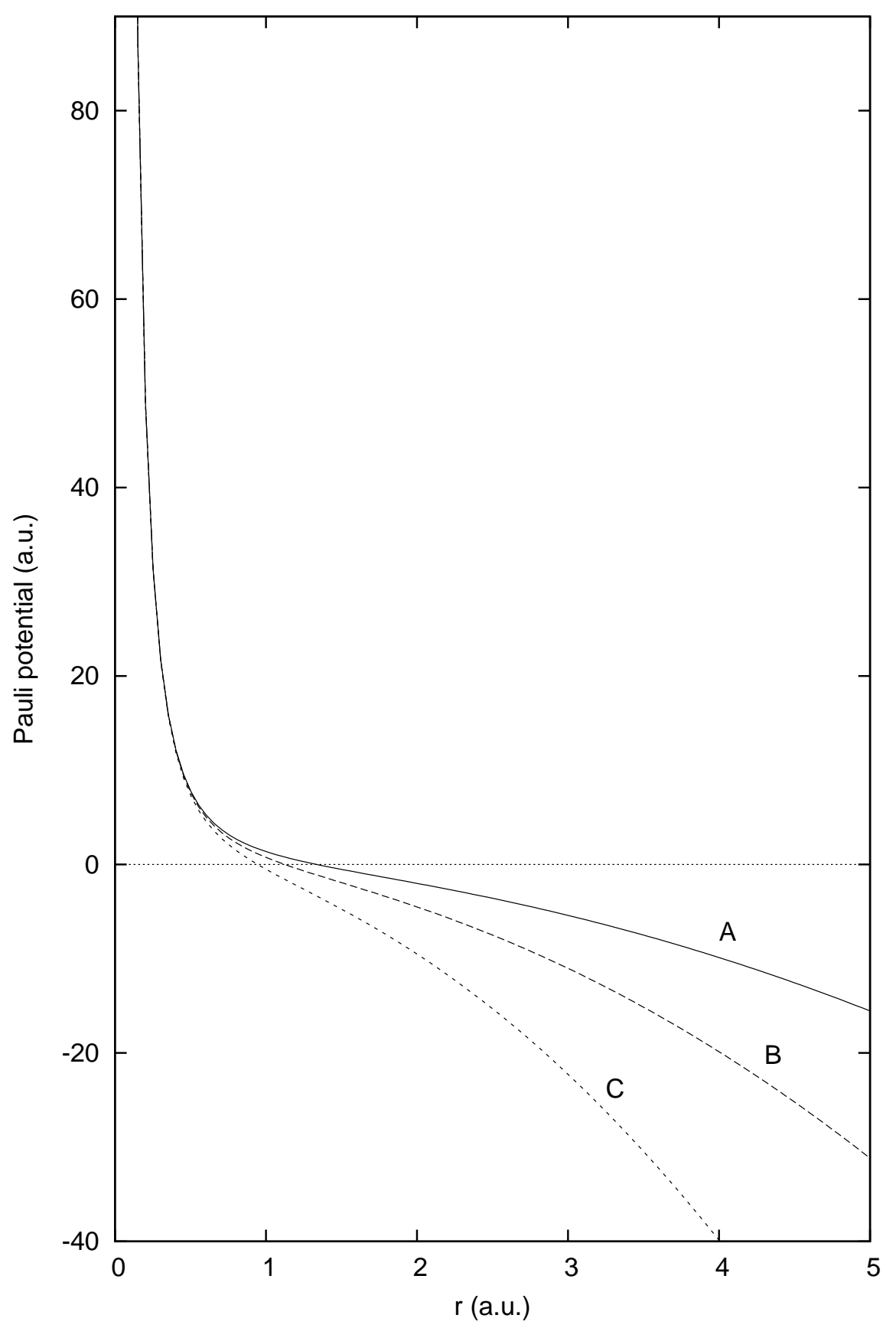

Figure 1: Plot of the Pauli potential derived from the pair density of the quintet state of four electrons interacting via harmonic potential energy $(1 / 2) \kappa r^{2}$ against interparticle separation $r$. Curves A, B and $\mathrm{C}$ refer, respectively, to the cases with $\kappa=0.5,1$ and 2 a.u. 